\title{
Jet Quenching Measurements at RHIC
}

\author{
Olga Barannikova ${ }^{1}$ \\ 1 University of Illinois at Chicago, \\ 845 W. Taylor St. M/C 273 Chicago IL 60607-7059 USA
}

Received on 4 December, 2006; revised version received on 8 March, 2007

\begin{abstract}
Experimental observation of jet quenching in ultra-relativistic heavy-ion collisions is one of the most remarkable discoveries at RHIC. High- $p_{T}$ hadron suppression, disappearance of back-to-back jets, and strong away-side modification at intermediate to low $p_{T}$ have provided us many insights into the matter created at RHIC. Particularly, angular correlations have become a powerful tool to study the QCD matter through its interactions with jets. Di-hadron correlations reveal significant broadening and softening of associated hadrons on the away side of a triggered high- $p_{T}$ particle. Many mechanisms have been proposed to accommodate the data, including the intriguing Mach cone shock waves in a thermalized hydrodynamic matter, which can be discriminated by 3-particle correlations. Here a brief overview of these remarkable experimental measurements on jet quenching is presented and implications of these measurements are discussed.
\end{abstract}

Keywords: RHIC; Heavy ion; Jet quenching; Energy loss

\section{INTRODUCTION}

The physics program at RHIC has enabled remarkable advances in the study of hot strongly interacting matter [1-4]. An extended reach in particle momentum at RHIC allowed for development of "hard probes" to study the behavior of the created medium that was difficult to access at lower collision energies. The hard penetrating probes are now one of the major experimental tools successfully explored at RHIC.

High $p_{T}$ hadrons are predominantly produced by initial hard-scattered partons. Partons and/or fragmented hadrons need finite time to escape the collision zone, during which the dense medium is formed. During this time they may lose energy via strong interactions with the medium, therefore providing the means to investigate its properties. The effect of energy loss due to these interactions with the medium is apparent in various experimental observations at RHIC. Two of the most striking early results showing large effects due to the traversed matter were $R_{A B}\left(p_{T}\right)$, the ratio of hadron yields in $\mathrm{A}+\mathrm{B}$ (either $\mathrm{Au}+\mathrm{Au}$ or $\mathrm{d}+\mathrm{Au}$ ) collisions to $p p$, corrected for geometric effects via scaling by number of binary (nucleonnucleon) collisions [5-7], and the disappearance of back-toback jets in the di-hadron azimuthal correlations [8].

The suppression of hadrons, apparent in inclusive charged hadron measurements as well as in identified hadrons (see, for example, Fig. 1), begins in the intermediate sector $\left(p_{T} \sim\right.$ $2 \mathrm{GeV} / c$ ), then saturates and remains constant as a function of $p_{T}$ throughout the entire hard region accessible in the experimental data. Conventional nuclear effects, such as nuclear shadowing of the parton distribution functions and initial state multiple scattering, cannot account for the observed suppression. Also, such suppression is not seen in $\mathrm{d}+\mathrm{Au}$ collisions, indicating that it is a final-state effect associated with the matter produced in $\mathrm{Au}+\mathrm{Au}$ events [9]. The results are consistent with the effects of parton energy loss in traversing dense medium, predicted before the data were available $[10,11]$. Those pQCD calculations can efficiently describe both the centrality- and $p_{T}$-dependence of the observed suppression. To reproduce the magnitude of the suppression, however, these calculations need to assume that the initial gluon density is more than an order of magnitude ( $\sim 30$ times) greater than that of cold, confined nuclear matter (or energy density of $\sim 100$ times higher) [12]. At the same time, a hadronic transport calculation [13] also leads to conclusion that the absorption of formed hadrons in the medium fails to account for the magnitude of the observed suppression. Recent experimental results of single inclusive measurements are discussed in greater details in [14].

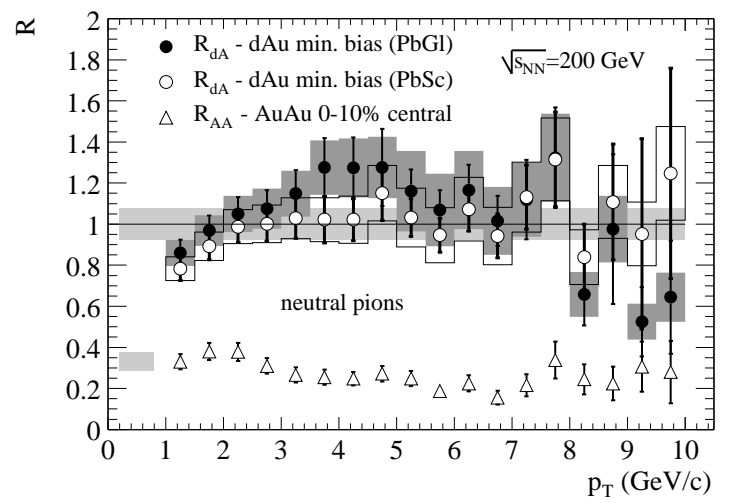

FIG. 1: PHENIX measurements [6] of nuclear modification factors $R_{A B}\left(p_{T}\right)$ for minimum bias $\mathrm{d}+\mathrm{Au}$ and central $\mathrm{Au}+\mathrm{Au}$ collisions illustrate the strong suppression of pion yields in central $\mathrm{Au}+\mathrm{Au}$ collisions, in comparison to the expectations from $p p$ collisions, scaled by the number of contributing binary collisions. No suppression is observed in the $\mathrm{d}+\mathrm{Au}$ data.

\section{DIRECT DI-JET OBSERVATION}

Di-hadron correlations provide additional means to probe the medium. They allow the experimental observation of the energy lost by partons in form of an excess of softer emerging hadrons. An analysis of azimuthal correlations between hard and soft hadrons [15] demonstrates that, in comparison 


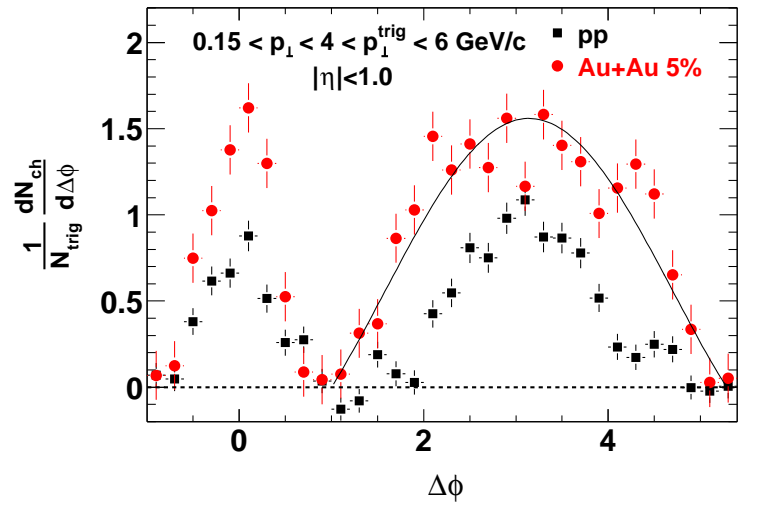

FIG. 2: Background-subtracted $\Delta \phi$ distributions for $p p$ and central $\mathrm{Au}+\mathrm{Au}$ data by STAR [15]. As seen in the plot, more soft associated hadrons are found per trigger particle in central $\mathrm{Au}+\mathrm{Au}$ than in pp, on both the near and away sides.

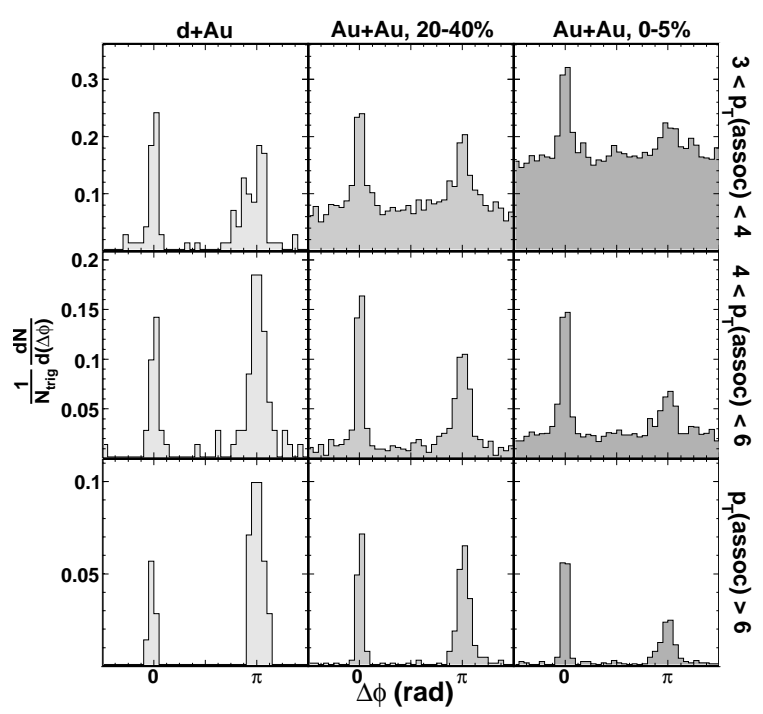

FIG. 3: STAR measurements of di-hadron azimuthal correlations associated with a charged hadron trigger with $8<p_{T}<15 \mathrm{GeV} / c$ for $200 \mathrm{GeV} \mathrm{Au+Au}$ and $\mathrm{d}+\mathrm{Au}$ collisions.

with $p p$ and peripheral $\mathrm{Au}+\mathrm{Au}$ collisions, the momentumbalancing hadrons, associated with a high- $p_{T}$ trigger hadron on away side, are greater in number, dispersed wider in azimuthal angle, and significantly softer (Fig. 2). These results indicate that particles from two different sources, initial hardscattered partons (or jets) and the bulk medium, have interacted intensively approaching to some degree equilibration with each other. This may in fact reflect a high degree of thermalization in the medium itself, a necessary condition for the formation of quark-gluon plasma. It has been found also that correlation strength is sensitive to the in-medium path length of the parton [16], confirming the jet-medium interaction as a source for the observed modifications.

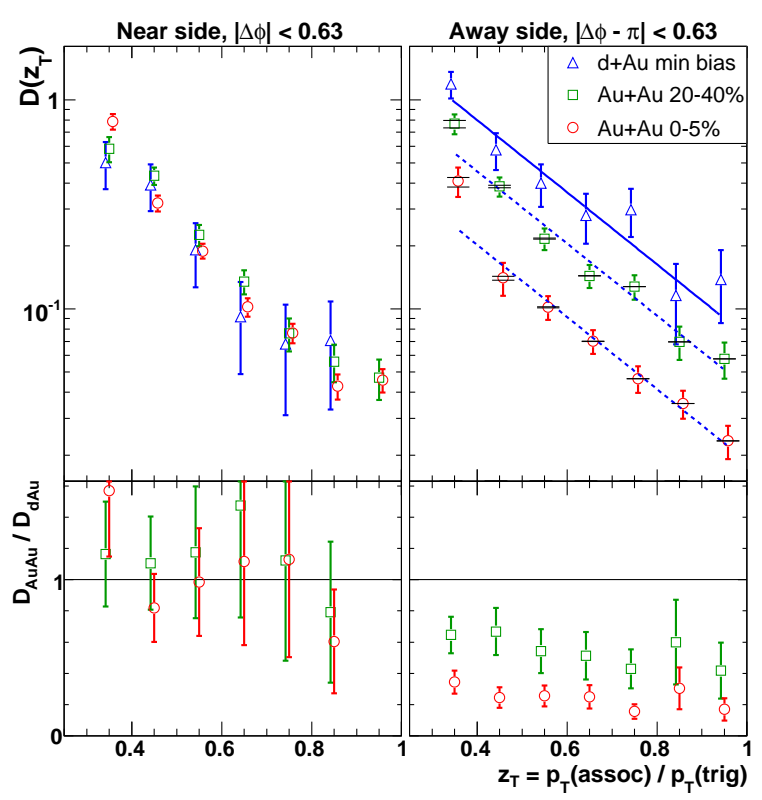

FIG. 4: Upper panels: trigger-normalized charged hadron fragmentation function $\mathrm{D}\left(z_{T}\right)$ with triggers $8<p_{T}<15 \mathrm{GeV} / c$, for near- (left) and away-side (right) correlations in $200 \mathrm{GeV} \mathrm{d}+\mathrm{Au}$ and $\mathrm{Au}+\mathrm{Au}$ collisions. Lower panels: ratio of $\mathrm{D}\left(z_{T}\right)$ for $\mathrm{Au}+\mathrm{Au}$ relative to $\mathrm{d}+\mathrm{Au}$.

With the increased statistics of experimental data, the dihadron azimuthal correlation studies have advanced substantially in recent years in terms of transverse momentum reach. A new effect, in contrast to the previously seen disappearance or modification of the away-side jet in central $\mathrm{Au}+\mathrm{Au}$ collisions, has been observed for an intermediate transverse momentum threshold. Di-hadron azimuthal correlations, in which the trigger and the associated particle are both from the hard sector, show clear jet-like peaks in both near- and away-sides (Fig. 3) [17]. Little modification is apparent in the shape of the peaks, but the height of the away-side peak shows a clear suppression in central $\mathrm{Au}+\mathrm{Au}$ collisions with respect to that in $\mathrm{d}+\mathrm{Au}$ collisions. Systematic studies of correlation strengths and shapes provided further insights about the properties of the matter formed at RHIC. An observed increase in the correlated yield on a trigger side with increasing trigger $p_{T}$ is expected in a jet fragmentation picture due to bias towards higher $E_{T}$ jets provided by higher $p_{T}$ trigger. Suppression of the away side peak is consistent with the observation of the hadron suppression in the single inclusive and measurements. However, as mentioned, no significant shape modifications of an away-side peak is observed at this high momentum range. This counter-intuitive observation is in contrast with a multiple scattering scenario, where one would expect that the strong suppression to be accompanied by a broadening of the away-side peak [18, 19].

No effects of the jet-medium interactions at high $p_{T}$ are observed in the transverse momentum distributions of near- and away-side associated hadrons. As seen in Fig. 4, the triggernormalized fragmentation function for both the near-side (left panels) and away-side distributions are similar over a broad 
range of $z_{T}=p_{T_{\text {assoc }}} / p_{T_{\text {trig }}}$ for $p p, \mathrm{~d}+\mathrm{Au}$ and $\mathrm{Au}+\mathrm{Au}$ colliding systems. Strong away-side hadron suppression at high $p_{T}$ that is not accompanied by modification of angular correlations or momentum distributions suggests in-medium partonic energy loss followed by in-vacuum fragmentation and provides challenges for many theoretical calculations.

\section{THREE-PARTICLE CORRELATIONS}

Although at RHIC jet fragmentation seem to be unaffected by the medium in a high energy limit, modifications of jet shapes and momentum distributions are quite significant in the intermediate and soft sectors. Not only are the correlated hadrons on the away side of the trigger particle found to be broadly distributed, their transverse momentum distribution is similar to that of the bulk, indicating partial thermalization of the jet products in the strongly interacting medium. The away-side broadening becomes increasingly prominent for the lower energy triggers. In fact, for some particular kinematic selections of correlated particles the overall broadening of the away-side distribution is accompanied by the developing of a dip at $\Delta \phi \approx \pi$ as illustrated in Fig. 5 by PHENIX and STAR measurements.
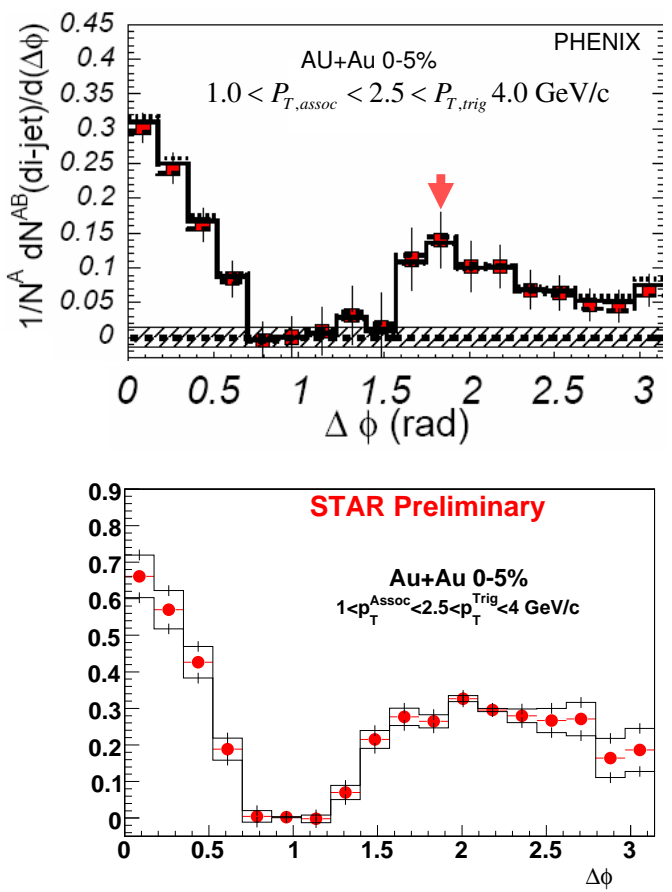

FIG. 5: $\Delta \phi$ correlations in central 5\% $\mathrm{Au}+\mathrm{Au}$ collisions from PHENIX [20] (up) and STAR [21] (down).

This novel shape of the away side distributions stirred many theoretical discussion and investigations. Various scenarios and different mechanisms of jet-medium interactions have been proposed to explain the experimental observations [2225]. In particular, shock wave induced conical flow ("sonic boom"), Čerenkov gluon radiation, and a "deflected jet" induced by interactions between the propagating partons and the flowing medium. Mach-cone shock waves in the hydrodynamic medium would be generated at a certain $p_{T^{-}}$ independent angle, determined by the speed of sound in the medium. On the other hand, Čerenkov gluon radiation would be characterized by a $p_{T}$-dependent angular spread of the double-peak structure. Detailed studies of three particle correlations as function of transverse momentum of correlated particles could help discriminating various energy loss scenarios. The importance of such investigations is recognized by both PHENIX and STAR experiments, which have recently reported preliminary results of three particle studies, employing slightly different analysis techniques.

PHENIX studies jet properties via three-particle correlation functions built by combining a high $p_{T}$ hadron in a transverse momentum range $2.5<p_{T}<4.0 \mathrm{GeV} / c$ with two lower $p_{T}$ (1.0 to $2.5 \mathrm{GeV} / c$ ) associated hadrons in the frame of reference, where $\mathrm{Z}$-axis is defined by the high $p_{T}$ particle momentum vector, and a two dimensional correlation function is obtained in $\theta^{*}, \Delta \phi^{*}$ space, where $\theta^{*}$ is the polar angle of one of the low $p_{T}$ particles and $\Delta \phi$ is the difference of azimuthal angles of the two associated particles) [26]. Fig. 6 shows the resulting correlation surface (for 20-40\% mid-central $\mathrm{Au}+\mathrm{Au}$ data) after removal of the background contributions [27].

The away-side jet in this correlation analysis show a broad minimum at $180^{\circ}$ for most of the $\Delta \phi^{*}$, and an apparent maximum (or quite sizable correlation) in this distribution at the away-side angle of $120^{\circ}$, that is intriguingly close to theoretically estimated Mach-cone angle, and has been interpreted as initial evidence for a sonic boom in hot QCD matter. Studies are underway to evaluate all possible background contributions to the measured correlation.

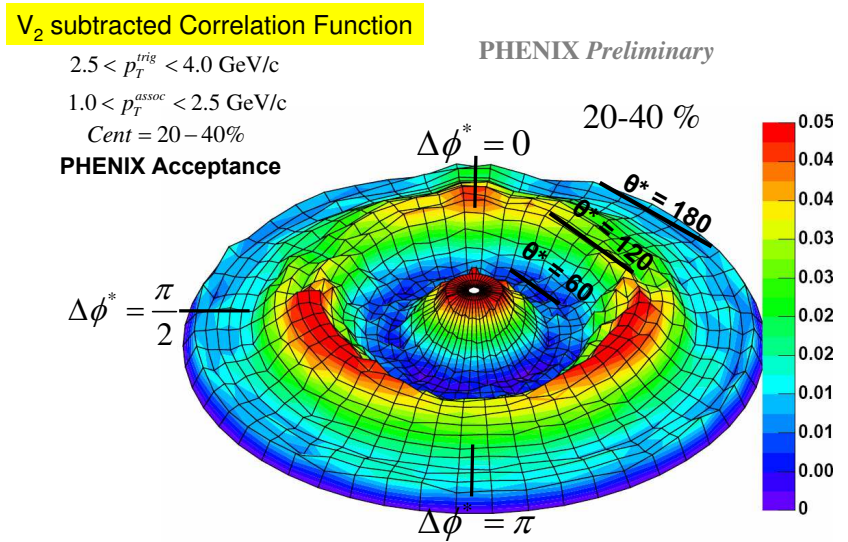

FIG. 6: Three particle correlation surface for charged hadrons detected in semi-central (20-40\%) Au+Au collisions within the PHENIX acceptance.

Preliminary STAR results on the three-particle correlation analysis [28, 29] are shown in Fig. 7. Here, for all three 


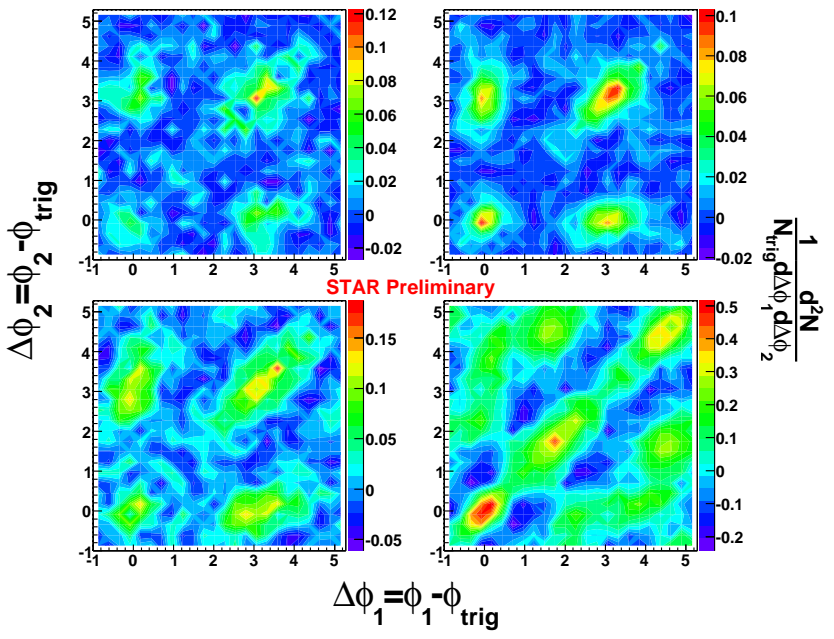

FIG. 7: Background subtracted 3-particle azimuthal correlations from STAR for pp (top left), d+Au (top right), Au+Au 50-80\% (bottom left), and ZDC triggered $0-12 \%$ central $\mathrm{Au}+\mathrm{Au}$ (bottom right).

systems studied $(\mathrm{Au}+\mathrm{Au}, \mathrm{d}+\mathrm{Au}$ and $p p$ collisions at 200 $\mathrm{GeV}$ ) the kinematic selection is slightly different than that of PHENIX selections: trigger particles are selected in the transverse momentum range of $3.0<p_{T}<4.0 \mathrm{GeV} / c$, and the correlation function is build with hadrons with transverse momentum between $1.0<p_{T}<2.0 \mathrm{GeV} / c$. The correlation surface reveals more details about the jet fragmentation and propagation through the medium. The elongation of awayside peak along the diagonal in the $\Delta \phi_{1}, \Delta \phi_{2}$ space is already observed in $p p$ and $\mathrm{d}+\mathrm{Au}$ data and is interpreted as possible effects of $k_{T}$ broadening. This elongation becomes more pronounced in $\mathrm{Au}+\mathrm{Au}$ collisions potentially indicating the presence of some additional mechanisms, such as for example deflected jets, contributing to shape of the observed correlation. Observation of off-diagonal peaks evident in more cen- tral $\mathrm{Au}+\mathrm{Au}$ data is consistent with conical flow or Čerenkov radiation scenarios. Further detailed studies of the precise location and the $p_{T}$ dependence of this off-diagonal structure should provide the discriminating power between the two scenarios.

\section{SUMMARY}

In summary, significant advances have been made in hard sector studies at RHIC by means of single- and di-hadron penetrating probes. The magnitudes of the measured suppressions of high $p_{T}$ yields and jet-like angular correlations in central $\mathrm{Au}+\mathrm{Au}$ collisions suggest that the initial energy density of the created medium is orders of magnitude larger than the normal nuclear density. Clear evidence of energy loss is apparent in both soft-hard and hard-hard correlations. Re-emerging of the away side jet at higher transverse momentum allows to investigate directly the features of jet fragmentation functions in heavy-ion collisions. Observation of unmodified associate distributions suggests that fragmentation time exceeds the medium traversal time of the leading parton. Recent results of energy loss studies via three-particle correlation techniques by both PHENIX and STAR yielded very suggestive results, with evidence for both presence of the deflected jets and conical emission, that seem to support a Mach-cone shock waves emission at RHIC. Studies are underway to evaluate all possible background contributions of those complex analyzes and finalize the results, so that the speed of sound and the equation of state for the medium created at RHIC could be extracted.

\section{Acknowledgments}

The author would like to thank the organizers for the kind invitation to participate in this conference. This work was supported by a grant from the US Department of Energy DEFG02-94ER40865.
[1] I. Arsene et al. (BRAHMS), Nucl. Phys. A 757, 1 (2005).

[2] K. Adcox et al. (PHENIX), Nucl. Phys. A 757, 184 (2005).

[3] B. B. Back et al. (PHOBOS), Nucl. Phys. A 757, 28 (2005).

[4] J. Adams et al. (STAR), Nucl. Phys. A 757, 102 (2005).

[5] I. Arsene et al. (BRAHMS), Phys. Rev. Lett. 91, 072301 (2003).

[6] S. Adler et al. (PHENIX), Phys. Rev. Lett. 91, 072303 (2003).

[7] B. Back et al. (PHOBOS), Phys. Rev. Lett. 91, 072302 (2003).

[8] J. Adams et al. (STAR), Phys. Rev. Lett. 91, 072304 (2003).

[9] J. Adams et al. (STAR), Phys. Rev. Lett. 91, 072304 (2003).

[10] I. Vitev and M. Gyulassy, Phys. Rev. Lett. 89, 252301 (2002).

[11] X.-N. Wang, nucl-th/0305010.

[12] X.N. Wang, Phys. Lett. B 595, 165 (2004).

[13] W. Cassing, K. Gallmeister, and C. Greiner, Nucl. Phys. A 735, 277 (2004).

[14] J. Lajoie, in these proceedings Braz. J. Phys. 37, 825 (2007).

[15] J. Adams et al. (STAR), Phys. Rev. Lett. 95, 152301 (2005).

[16] J. Adams et al. (STAR), Phys. Rev. Lett. 93, 252301 (2004).
[17] J. Adams et al. (STAR), Phys. Rev. Lett. 97, 162301 (2006).

[18] R. Baier et al., Nucl. Phys. B 484, 265 (1997).

[19] I. Vitev, Phys. Lett. B 630, 78 (2005).

[20] S. Adler et al. (PHENIX), Phys. Rev. Lett. 97, 052301 (2006).

[21] J. Ulery (STAR), Nucl. Phys. A 774, 581 (2006).

[22] H. Stoecker, Nucl. Phys. A 750, 121 (2005)

[23] J. Casalderrey-Solana, E. V. Shuryak and D. Teaney, J. Phys. Conf. Ser. 27, 22 (2005); Nucl. Phys. A 774, 577 (2006).

[24] J. Ruppert and B. Muller, Phys. Lett. B 618, 123 (2005).

[25] V. Koch, A. Majumder, and X. N. Wang, Phys. Rev. Lett. 96, 172302 (2006).

[26] N. Ajitanand (PHENIX), Nucl. Phys. A 783, 519 (2007).

[27] N. Ajitanand et al., Phys. Rev. C 72, 011902 (2005).

[28] F. Wang, nucl-ex/0610027 (2006).

[29] J. Ulery, nucl-ex/0608046 (2006). 